\title{
Novel cancer therapeutics could target ion channels
}
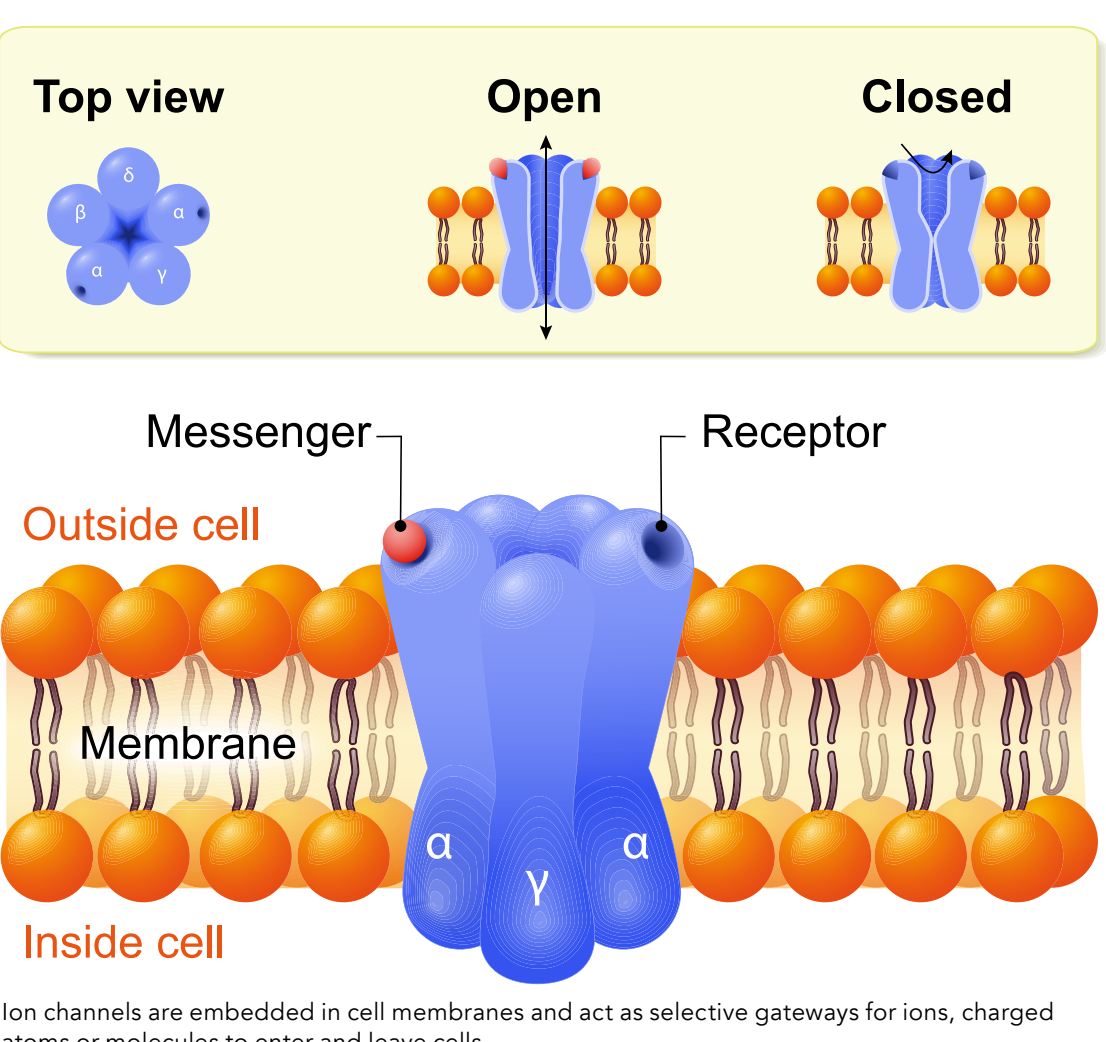

\section{POTASSIUM CHANNELS}

THE ROLE OF

Potassium ion channels are vital in

of cells using energy. The acquisition

of motility is essential in the metastasis

of cancer (i.e. when cancer cells

travel from one part of the body

to another to form new tumours.

Specifically, potassium channels

regulate the ability of a cell to switch

from an epithelial state, in which they

are adhered together and stuck to

a basement membrane, to what's

called a mesenchymal state, in which

they lose this adhesion. This gives

cancer cells enhanced migratory and

invasive capabilities. As various cancers

abnormally express certain potassium

critical factors in cancer biology.

Upon investigating this further, Dr Gentile found that several of these channels play a pivotal role in controlling some of the key traits of cancers. These included cancer cell proliferation and tumour growth, tumour metastasis and cancer cell survival. As such, a drug that could target these channels and affect their function has the potential to arrest tumour growth and metastasis, effectively stopping the development of cancer.
A drug that could target these channels and affect their function has the potential to arrest tumour growth and metastasis.

subunits of either the Kirb.1 or Kirb.2 proteins bound to a receptor, either is already a target for several existing, FDA-approved drugs, including glibenclamide, an anti-diabetic medication that targets the Kirb.1/ hypertensive and thoxidi, an antiof different Kirb subunits with either the SUR1 or SUR2 receptor grants the drug tissue specificity, meaning that a drug targeting Kir6 2/SUR2 will not affect tissues with the Kirb.1/SUR1 channel, effectively eliminating any side effects.

Gynaecological cancer cells have been found to express Kirb.2/SUR2 and so can be targeted with minoxidil, which activates the channel. Dr Gentile found that the use of minoxidil to treat ovaria cancer arrested tumour growth by altering the metabolic state of the cells through mitochondrial disruption and SUR1 or SUR2. The Kirb/SUR channel Kirb.2/SUR2 channel. The expressio minoxidil activates a cell death pathway, growth. The same effect was seen endometria

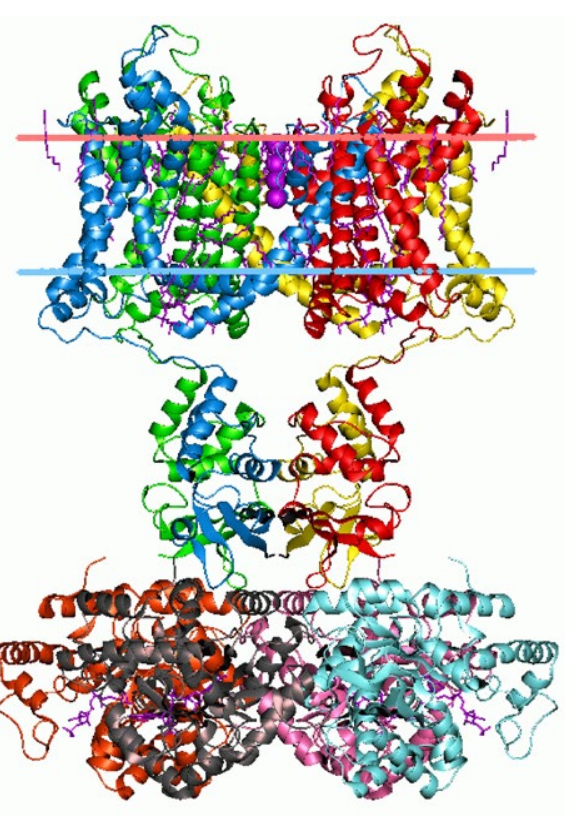

Potassium ion channels are vital for regulating
the movement of cells. resulting in greatly reduced tumour
The tissue specificity afforded by minoxidil did not induce meant that even in high doses. This overcomes the issues of dose-limiting toxicity that surround current treatments for gynaecological cancers, which can both increase morbidity and limit the ability to deliver the optimal therapeutic dose.

Excitingly, these findings suggest that repurposing of existing potassium channel activators could represent a new and, most importantly, safe gynaecolo approach to treating gynaecological cancers that is both lowcost and readily available. As minoxidi is also already FDA-approved, it has the pom tra to unic.

\section{BATTLING BREAST CANCERS}

It's not only gynaecological cancers that Dr Gentile has also explored their role in the biology of breast cancer.

In breast cancer, the clinical outcome is heavily influenced by the expression of proteins associated with the oestrogendependent signalling and the HER2 signalling pathway. Therapeutics that tathet either of these signalling pathways types of breast cancer called oestrogenreceptor positive or HER2 overexpressing cancers but are ineffective against what's uses neither of these signalling pathways.

However, high levels of expression of the gene that encodes for the potassium channel Kv11.1 is associated with good prognosis in oestrogenthe early stages of cancers, ex. In of Kv11. 1 is associated with the transition into the mesenchymal state, allowing the cells to migrate. As such, uncontrolled gain or loss in Kv11.1 activity is linked to tumour progression.

Dr Gentile investigated the effect of activating the Kv11.1 channel on cancer development and found that it significantly reduced the metastatic spread of tumours. Use of a Kv11, inhibited cell motilit by $\mathrm{PS} 643$ inhibited cell motility by preventing the
transition from epithelial to mesenchymal 


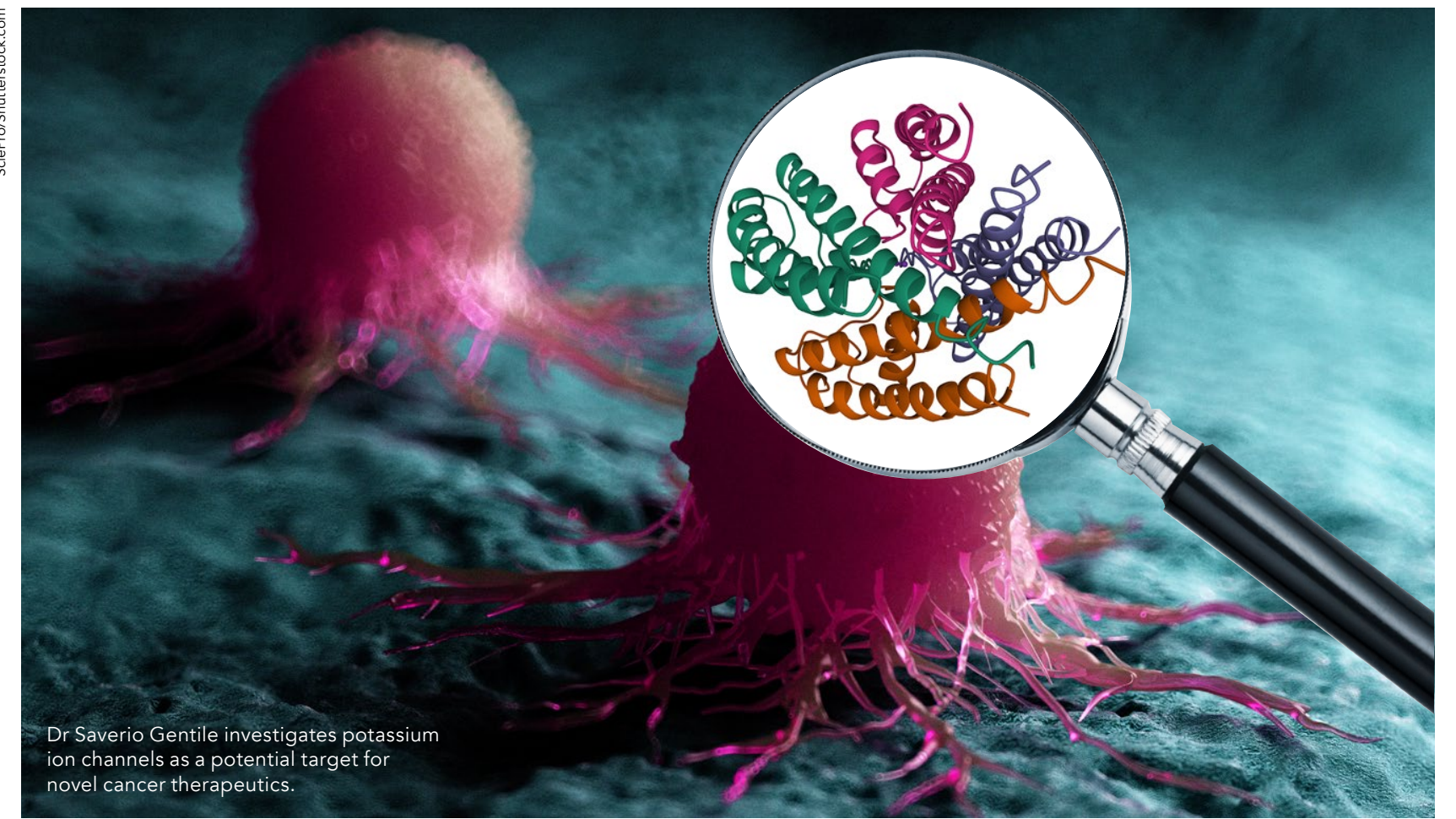

states, thus stopping the cells from migrating and invading other tissues. It was also found to reduce cancer cell 'stemness', which is the ability of the differentiate into different cell types.

the cell cycle, preventing them from replicating. This arrest of the cell cycle increased the level of proteins in the cell associated with senescence, a state in which cells stop dividing due to stress factors like DNA damage. However, senescence is also thought to be a natural anti-tumour mechanism and thus inducing this state in NSI643 works of a molecule called beta-catenin, which is found mostly at when cells are when cells are adhered together nucleus to stimulate cell migration by activating signalling pathway. signalling pathway.
NS1643 inhibits the degradation

of beta-catenin and promotes its localisation to the cell membran preventing the cells from migrating Remarkably, the Kv11.1 channel is not expressed in normal, healthy breast cells, so the use of NS1643 is selective against cancerous cells. Importantly, these types of drugs do not alter heart perfornance suggesting that a potential therapeutic approach is

NS1643 was also found to irreversibly stop cancer cells in one phase of ion channels. proliferating. Additionally, senescent cells are more susceptible to the effects of traditional chemotherapy drugs. As such, activating potassium channels may work both as an anticancer therapy in itself but also as an adjuvant therapy, making traditional

MORE THAN JUST POTASSIUM Whilst potassium ion channels have been the focus of Dr Gentile's recent research, he has also suggested that chemotherapy more effective.

\section{偖 . 政 然}

\section{Behind the Research}

\section{Dr Saverio Gentile}

E: sgentile@uic

also have therapeutic effects in other cancer types by reviewing similar research literature on the subject. For example, inhibiting calcium ion channels with small molecule inhibitors like mibefradil has been found to reduce cell proliferation in prostat cancer by inducing cell death, and a similar result was seen in gliomas targeted with activators called bufadienolides. In total, eleven types showed types showed
reduced cell proliferation proliferation
through targeted through targe
activation or
inhibition of

\section{Research Objectives}

Dr Gentile developed a successful technical approach allowing the dissection of ion channel-dependent research explores ion channel-dependent involvement in disease.

\section{Detail}

Address

Saverio Gentile, University of Illinois at Chicago

Department of Medici

Division of Hematology and Oncology

840 S Wood Street

Chicago, IL 60612 USA

Bio

Dr Gentile earned a PhD in neuroscience and studied ion channels at the NIEHS/NIH and Duke university. In 2009 he started his lab in Pharmacology departmen at Loyola University, Chicago. In 2018 Dr Gentile

moved to the Department of Medicine, University Chino at Chicago where he directs the Lab of lon Channels in Cancer.

Funding

Defertment of Defence Breast Cancer Breakthrough Award; The Cronk Foundation; The Christl Burgess Memorial Fund for ovarian cancer research; Cures Within Reach

\section{Collaborators}

- Kent Hoskins (Breast cancer physician) - Ronald K. Potkul (Ovarian cancer physician)

With treatments for advanced-stage gynaecological and triple-negative breast cancers still severely lacking in availability, potassium channels could present a novel target for cancer therapeutics. Repurposing existing potassium channel activators has the potential to provide a new, safe and strategy and increr pharmacological strategy and increase the efficacy of

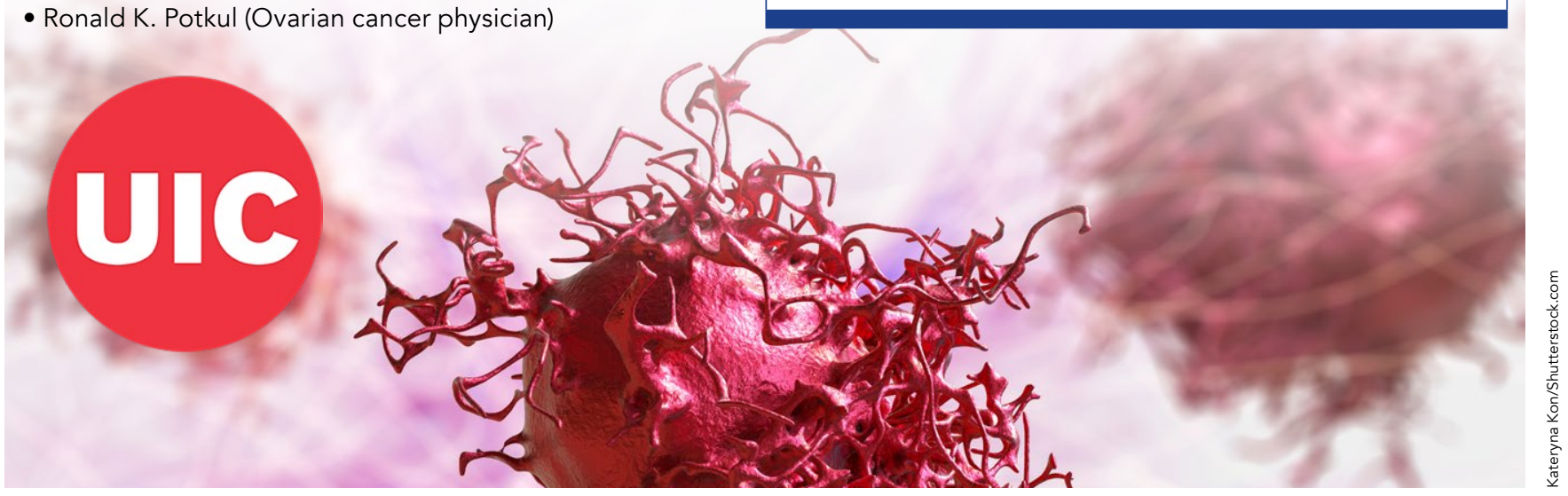

\section{References}

Gentile, S. \& Lansu, K. (2013). Potassium channel activation inhibits proliferation of breast cancer cells by activating a senescence program. Cell Death and Disease [Online]. e652. https://www.nature.com/articles/cddis2013174

Gentile, S. et al. (2015) Voltage-Gated lon Channels in Cancer Cell Proliferation. Cancers. 7, pp. 849-875.

Gentile, S. et al. (2019) Potassium channel activity controls breast cancer metastasis by affecting $\beta$-catenin signaling nature.com/articles/s41419-019-1429-0

Gentile, S. et al. (2020). Repurposing Kirb/SUR2 Channel Activator Minoxidil to Arrests Growth of Gynecological Cancers. Front. Pharmacol. [Online]. 11, Article fphar.2020.00577/full

\section{Personal Response}

What are the next steps in getting drugs like minoxidil officially repurposed into new cancer reatments?

II The next step is to test the effects of minoxid through a clinical trial. We are already preparing in testing whether our approach can potentiate the anticancer effects of therapeutic agents that are 